\title{
A gene variant near $A T M$ is significantly associated with metformin treatment response in type 2 diabetes: a replication and meta-analysis of five cohorts
}

\author{
N. van Leeuwen • G. Nijpels • M. L. Becker • \\ H. Deshmukh • K. Zhou • B. H. C. Stricker • \\ A. G. Uitterlinden • A. Hofman • E. van 't Riet • \\ C. N. A. Palmer • B. Guigas • P. E. Slagboom • \\ P. Durrington • R. A. Calle • A. Neil • G. Hitman • \\ S. J. Livingstone $\cdot$ H. Colhoun $\cdot$ R. R. Holman • \\ M. I. McCarthy • J. M. Dekker • L. M. 't Hart • \\ E. R. Pearson
}

Received: 20 November 2011 / Accepted: 28 February 2012 / Published online: 28 March 2012

(C) The Author(s) 2012. This article is published with open access at Springerlink.com

\begin{abstract}
Aims/hypothesis In this study we aimed to replicate the previously reported association between the glycaemic response to metformin and the SNP rs11212617 at a locus that includes the ataxia telangiectasia mutated (ATM) gene in multiple additional populations.
\end{abstract}

L. M. 't Hart and E. R. Pearson contributed equally to this study.

Electronic supplementary material The online version of this article (doi:10.1007/s00125-012-2537-x) contains peer-reviewed but unedited supplementary material, which is available to authorised users.

N. van Leeuwen · B. Guigas · L. M. 't Hart

Department of Molecular Cell Biology,

Leiden University Medical Center,

Leiden, the Netherlands

G. Nijpels · E. van 't Riet · J. M. Dekker

EMGO Institute for Health and Care Research,

VU University Medical Center,

Amsterdam, the Netherlands

G. Nijpels

Department of General Practice, VU University Medical Center, Amsterdam, the Netherlands

M. L. Becker - B. H. C. Stricker - A. G. Uitterlinden - A. Hofman Department of Epidemiology, Erasmus Medical Center,

Rotterdam, the Netherlands

M. L. Becker

Department of Hospital Pharmacy, Erasmus Medical Center,

Rotterdam, the Netherlands
Methods Incident users of metformin selected from the Diabetes Care System West-Friesland (DCS, $n=929)$ and the Rotterdam Study $(n=182)$ from the Netherlands, and the CARDS Trial $(n=254)$ from the UK were genotyped for rs11212617 and tested for an association with both $\mathrm{HbA}_{1 \mathrm{c}}$ reduction and treatment success, defined as the ability to

H. Deshmukh • K. Zhou • C. N. A. Palmer - S. J. Livingstone •

H. Colhoun $\cdot$ E. R. Pearson $(\triangle)$

University of Dundee, Medical Research Institute,

Dundee DD1 9SY, UK

e-mail: e.z.pearson@dundee.ac.uk

E. van 't Riet $\cdot$ J. M. Dekker

Department of Epidemiology and Biostatistics,

VU University Medical Center,

Amsterdam, the Netherlands

P. E. Slagboom • L. M. 't Hart $(\bowtie)$

Leiden University Medical Center, Molecular Epidemiology, Postzone S5-P, PO box 9600, 2300RC Leiden, the Netherlands e-mail: 1.m.t_hart@lumc.nl

P. E. Slagboom

Netherlands Consortium for Healthy Ageing, the Netherlands

URL: www.healthy-ageing.nl 
reach the treatment target of an $\mathrm{HbA}_{1 \mathrm{c}} \leq 7 \%(53 \mathrm{mmol} / \mathrm{mol})$. Finally, a meta-analysis including data from literature was performed.

Results In the DCS cohort, we observed an association between rs11212617 genotype and treatment success on metformin (OR 1.27, 95\% CI 1.03, 1.58, $p=0.028$ ); in the smaller Rotterdam Study cohort, a numerically similar but non-significant trend was observed (OR $1.45,95 \%$ CI 0.87 , 2.39, $p=0.15$ ); while in the CARDS cohort there was no significant association. In meta-analyses of these three cohorts separately or combined with the previously published cohorts, rs11212617 genotype is associated with metformin treatment success (OR 1.24, 95\% CI 1.04, 1.49, $p=0.016$ and OR $1.25,95 \%$ CI $1.33,1.38, p=7.8 \times 10^{-6}$, respectively).

Conclusions/interpretation A gene variant near ATM is significantly associated with metformin treatment response in type 2 diabetic patients from the Netherlands and the UK. This is the first robustly replicated common susceptibility locus found to be associated with metformin treatment response.

Keywords Genetics of type 2 diabetes $\cdot$ Human . Meta-analysis · Metformin · Oral pharmacological agents

\begin{tabular}{|c|c|}
\hline Abbrev & \\
\hline AMPK & AMP-activated protein kinase \\
\hline ATM & Ataxia telangiectasia mutated \\
\hline
\end{tabular}

\section{P. Durrington}

University of Manchester, School of Medicine,

Manchester, UK

R. A. Calle

Pfizer Ltd,

New York, NY, USA

A. Neil

Department of Primary Health Care, University of Oxford,

Oxford, UK

\section{G. Hitman}

Barts and the London School of Medicine and Dentistry, Queen Mary University of London,

London, UK

R. R. Holman • M. I. McCarthy

Oxford NIHR Biomedical Research Centre, Churchill Hospital, Oxford, UK

R. R. Holman • M. I. McCarthy

Oxford Centre for Diabetes, Endocrinology and Metabolism, University of Oxford, Churchill Hospital,

Oxford, UK

M. I. McCarthy

Wellcome Trust Centre for Human Genetics, University of Oxford, Oxford, UK
CARDS Collaborative Atorvastatin Diabetes Study

DCS Diabetes Care System West-Friesland

DPP Diabetes Prevention Program

GoDARTS Genetics of Diabetes Audit and Research Tayside

HWE Hardy-Weinberg equilibrium

LD Linkage disequilibrium

SNP Single-nucleotide polymorphism

UKPDS UK Prospective Diabetes Study

\section{Introduction}

Metformin is the first-choice treatment for type 2 diabetes [1]. It mainly improves fasting glucose levels by decreasing hepatic glucose production by inhibiting the mitochondrial respiratory-chain complex [2,3], leading to activation of the AMP-activated protein kinase (AMPK) [4] through increased cellular AMP [5]. Activation of the AMPK pathways was thought to be an important underlying mechanism involved in the inhibition of hepatic gluconeogenesis by metformin [6]; however, AMPK-independent glucoselowering effects of metformin have been described recently [7], and therefore the exact molecular mechanism of action remains unclear.

Our recent genome-wide association study revealed that the single-nucleotide polymorphism (SNP) rs11212617, located near the ataxia telangiectasia mutated (ATM) gene, is associated with metformin treatment response [8]. It has been shown in UK participants with type 2 diabetes mellitus from the GoDARTS (Genetics of Diabetes Audit and Research Tayside) and UKPDS (UK Prospective Diabetes Study) cohorts $(n=3,920)$ that the minor $\mathrm{C}$ allele of rs11212617 is associated with two successful treatment outcomes: (1) the ability to achieve $\mathrm{HbA}_{1 \mathrm{c}}$ values $\leq 7 \%$ (53 mmol/mol); (2) lower $\mathrm{HbA}_{1 \mathrm{c}}$ when analysed as a quantitative trait [8]. rs11212617 is located in a large linkage disequilibrium (LD) block that includes other genes as well; however, ATM is the most likely candidate to be involved $[9,10]$.

In the Diabetes Prevention Program (DPP, $n=2,981$ ), there was no evidence of an association between rs 11212617 and the effect of metformin in delaying disease progression from impaired glucose tolerance to diabetes (CC vs AA, HR 1.22) [95\% CI 0.86, 1.74] $p=0.27$ ) [11]. Lack of replication is often caused by the use of different phenotypes. As the progression to diabetes phenotype differs markedly from the type 2 diabetes mellitus phenotype, we have attempted to replicate our original finding in three independent cohorts using the identical phenotypic definition to that used in the original discovery. For this study, we used the Diabetes Care System West-Friesland (DCS) [12] 
and the Rotterdam Study [13] from the Netherlands, and the multi-centre CARDS (Collaborative Atorvastatin Diabetes Study) Trial [14] from the UK. Data were analysed in the individual cohorts, separately and combined in a meta-analysis. Finally an additional meta-analysis was performed using these new cohorts combined with the previously described GoDARTS and UKPDS stage 2 replication cohorts [8].

\section{Methods}

Study cohorts and participants Inclusion criteria for all cohorts were: white ethnicity; continuous metformin treatment for at least 6 months; a pretreatment $\mathrm{HbA}_{1 \mathrm{c}}$ value measured within 6 months of the start of metformin and at least one measurement within the 18 months after; no other glucose-lowering medication prescription except stable sulfonylurea treatment before and during metformin treatment. Ethics approval was obtained from the Medical Ethics Committee of the VU Vrije Universiteit Medical Center for the DCS, the Erasmus Medical Center for the Rotterdam Study, and for CARDS each centre obtained local research ethics committee approval after approval from the Multi-centre Research Ethics Committee. All participants gave written informed consent.
Diabetes Care System West-Friesland The DCS started in 1996 and is a diabetes management model which aims to improve diabetes care by coordinating the different types of diabetes care and to improve patient empowerment by providing education. Patients from the West-Friesland region in the Netherlands visit a local DCS centre once a year for a medical examination; therefore longitudinal information on medical history, drug use and drug response is available for all patients $(n=5,424)$ [12]. Metformin response could be defined in 929 white patients, and their characteristics are shown in Table 1.

Patients were genotyped for rs11212617 with a Taqman assay (Assay ID C_1314213; Applied Biosystems, Nieuwerkerk a/d IJssel, the Netherlands) according to the manufacturer's protocol. The call rate was $97.6 \%$, and there was no deviation from Hardy-Weinberg equilibrium (HWE; $p=0.46$ ).

Rotterdam Study The Rotterdam Study is a prospective population-based cohort study of 10,994 whites aged 55 years and older in Rotterdam, the Netherlands. Patients were invited between 1990 and 1999 and have been followed since then. The aim of the study was to investigate determinants of chronic and disabling diseases [13]. Medication prescription data were available for nearly all participants. Metformin response could be defined in 182 patients, and their characteristics are shown in Table 1.

Participants were genotyped for rs609261, which is in high LD with rs $11212617\left(r^{2}=1.0\right.$, HAPMAP, CEU) using

Table 1 Characteristics of the cohorts included in this study

\begin{tabular}{|c|c|c|c|}
\hline Characteristic & DCS & Rotterdam Study & CARDS \\
\hline$n$ & 929 & 182 & 254 \\
\hline Age (years) & $63.4 \pm 10.0$ & $74.2 \pm 8.1$ & $61.4 \pm 8.8$ \\
\hline Male $(\%)$ & 56.4 & 45.1 & 69.0 \\
\hline BMI & $30.1 \pm 4.9$ & NA & NA \\
\hline Baseline $\mathrm{HbA}_{1 \mathrm{c}}(\%)$ & $6.7 \pm 1.0$ & $8.3 \pm 1.5$ & $8.7 \pm 1.4$ \\
\hline Baseline $\mathrm{HbA}_{1 \mathrm{c}}(\mathrm{mmol} / \mathrm{mol})$ & $50 \pm 11$ & $67 \pm 16$ & $72 \pm 15$ \\
\hline Adherence & NA & $88.4 \pm 14.7$ & NA \\
\hline eGFR & $91.8 \pm 36.1$ & NA & NA \\
\hline Responders (\%) & 67.1 & 57.7 & 52.0 \\
\hline Metformin monotherapy (\%) & 58.8 & 35.7 & 31.0 \\
\hline Genotype frequency rs11212617; AA/AC/CC (\%) & $32.5 / 47.1 / 20.4$ & $32.4 / 46.7 / 20.9$ & $32.3 / 44.4 / 23.2$ \\
\hline Quality $^{\text {a }}$ (selection, comparability, exposure) & $* * * *, * \mathrm{~b}, * * *$ & $* * * *, * \mathrm{~b}, * * *$ & $* * * *, * \mathrm{~b}, * * *$ \\
\hline
\end{tabular}

Data are means \pm SD or $n(\%)$

${ }^{a}$ Quality of the studies assessed with the Newcastle-Ottawa Assessment Scale (www.ohri.ca/programs/clinical_epidemiology/oxford.asp); the maximum scores for selection, comparability and exposure are $* * * *, * *$ and $* * *$, respectively. GoDARTS and UKPS are not represented in this table and both scored the maximum of $* * *, * *$ and $* * *$

${ }^{\mathrm{b}}$ The Rotterdam Study and CARDS lacked the covariates BMI and eGFR in the analysis, and the DCS lacked the covariate adherence in the analysis, therefore these studies only scored one star for comparability

eGFR, estimated glomular filtration rate calculated with the Cockcroft-Gault formula; NA, not available 
the Illumina $550 \mathrm{k} \mathrm{SNP}$ array according to the manufacturer's instructions. Quality controls were as described previously [15]. The call rate was $99.9 \%$, and there was no deviation from HWE $(p=0.75)$.

CARDS CARDS is a multicentre, placebo-controlled, double-blind study that enrolled 2,838 white men and women between 40 and 75 years of age and randomised them to receive $10 \mathrm{mg} /$ day of atorvastatin or placebo [14]. Patients had type 2 diabetes as well as one other risk factor for coronary heart disease. CARDS was carried out in 132 centres in the UK and Ireland. Metformin response as defined above could be characterised in 254 patients, and their characteristics are shown in Table 1.

DNA was genotyped at Perlegen Sciences (Mountain View, CA, USA) using a proprietary SNP set comprising 599164 SNPs. The call rate for rs 11212617 was $99 \%$, and there was no deviation from HWE $(p=0.1)$.

Statistical analysis Two different outcomes were used to measure the metformin treatment response: (1) the ability to reach the treatment target of $\mathrm{HbA}_{1 \mathrm{c}} \leq 7 \%(53 \mathrm{mmol} / \mathrm{mol})$, analysed with logistic regression; (2) the decrease in $\mathrm{HbA}_{1 \mathrm{c}}$ achieved, analysed as a quantitative trait with linear regression. In each cohort, the total group of metformin users was analysed, but also a separate analysis was performed on patients starting metformin monotherapy or those starting dual therapy (where metformin is added to stable sulfonylurea treatment). Furthermore, two separate analyses were performed, the first on the total patient group regardless of the baseline $\mathrm{HbA}_{1 \mathrm{c}}$, and the second using only individuals with a baseline $\mathrm{HbA}_{1 \mathrm{c}}>7 \%(53 \mathrm{mmol} / \mathrm{mol})$. The definition of the endpoints and covariates was similar to the original publication [8]. The covariates that were included in both the logistic and linear regression analyses were: baseline $\mathrm{HbA}_{1 \mathrm{c}}$, time between baseline $\mathrm{HbA}_{1 \mathrm{c}}$ and metformin start date, drug adherence, daily dose and creatinine clearance, if measured in the cohort. In all cohorts with genome-wide association study data available, principal component analysis was used to exclude participants of non-white origin. A priori power calculations using Quanto software (http:// hydra.usc.edu/gxe) based on the ORs obtained in our original publication ( 1.35 or 1.25 when only stage 2 replication data are included) showed that we had respectively $86 \%$ and $61 \%$ power in the DCS, $29 \%$ and $18 \%$ in the Rotterdam Study, and $39 \%$ and $24 \%$ in CARDS $(\alpha=0.05)$. Data were considered to be significant at $p<0.05$.

Meta-analysis The three cohorts described above were included in the meta-analyses of treatment success and treatment $\mathrm{HbA}_{1 \mathrm{c}}$. To assess the overall robustness of the association, a second set of meta-analyses was performed by also including the stage 2 replication cohorts previously described by Zhou et al: GoDARTS $(n=1,965)$ and UKPDS $(n=1,113)$. To avoid an overestimation of the effect of the SNP, the initial GoDARTS discovery cohort, which showed a very strong and highly significant association with treatment success (OR 1.64; 95\% CI 1.37, 1.99), was not included. The DPP Study was not included because of the substantial nature of the difference in the phenotype [11].

For meta-analysis, a fixed-effects model was used. The inconsistency index $I^{2}$ was used to assess between-study heterogeneity. To assess bias, funnel plots were generated, and Egger and Harbord tests were performed for, respectively, the linear and the logistic model. The meta-analysis was performed using Comprehensive Meta-analysis software (Biostat, Englewood, NJ, USA) and Stata software version 11.2 (Stata, College Station, TX, USA).

\section{Results}

The minor allele and genotype frequencies of the SNP, rs11212617, were similar in the three new cohorts $(p>0.2)$ and consistent with those reported for the GoDARTS and UKPDS cohorts. The minor allele frequency was 0.44 , and the genotype frequencies are shown in Table 1.

In the DCS cohort, including both patients receiving monotherapy and patients receiving dual therapy, we observed, as in our original publication, an association between the minor $\mathrm{C}$ allele of rs11212617 and treatment success on metformin (OR 1.27 [95\% 1.03, 1.58], $p=0.028$; Table 2). In the Rotterdam Study, we observed a similar but non-significant trend (OR 1.45 [95\% CI 0.87 , 2.39], $p=0.15$ ), but this was not seen in the CARDS cohort (OR 1.03 [95\% CI 0.68, 1.57], $p=0.86$ ). In all three cohorts, the effect of rs11212617 was larger in those receiving monotherapy compared with dual therapy (Table 2). In the analysis of on-treatment $\mathrm{HbA}_{1 \mathrm{c}}$ as a quantitative trait, there was no significant effect on $\mathrm{HbA}_{1 c}$ in either the DCS or the Rotterdam Study cohort. The only significant association was observed in the dual therapy group of the CARDS cohort, where the $\mathrm{C}$ allele was associated with highertreatment $\mathrm{HbA}_{1 \mathrm{c}}$ (Table 3). There was no association between $\mathrm{rs} 11212617$ genotype and baseline $\mathrm{HbA}_{1 \mathrm{c}}$ in any of the cohorts (data not shown).

In the meta-analysis of the three cohorts, there was an increase in the odds of treatment success with the presence of the $\mathrm{C}$ allele (OR 1.24, $p=0.016$ ), in the whole group and where restricted to those starting metformin monotherapy alone (OR 1.38, $p=0.015$ ). These results are consistent in size and direction with our previous publication, such that a combined meta-analysis including the GoDARTS and UKPDS stage 2 replication datasets from that publication resulted in a combined OR of 1.25 (95\% CI 1.13, 1.38), $p=7.8 \times 10^{-6} ; I^{2}=0.0 \%, p=0.74 ; p_{\text {Harbord }}=0.505$ (Table 2 , 
Table 2 Logistic regression for the ability to reach the metformin treatment target of $\mathrm{HbA}_{1 \mathrm{c}}<7 \%(53 \mathrm{mmol} / \mathrm{mol})$ according to the ATM rs11212617 genotype

Patients are included regardless of their baseline $\mathrm{HbA}_{1 \mathrm{c}}$. Additive logistic regression models were used to calculate the C-allelic OR in each cohort. Covariates included were baseline $\mathrm{HbA}_{1 \mathrm{c}}$, baseline gap (except DCS), daily dose, drug adherence (except for DCS and CARDS), and eGFR (except for CARDS and Rotterdam Study). In the meta-analysis, a fixed-effects model was used

Table 3 Linear regression for treatment $\mathrm{HbA}_{1 \mathrm{c}}$ per $A T M$ allele

Patients are included regardless of their baseline $\mathrm{HbA}_{1 \mathrm{c}}$. An additive linear regression model was used to calculate the per $\mathrm{C}$ allele change in treatment $\mathrm{HbA}_{1 \mathrm{c}}(\%)$. Covariates included were baseline $\mathrm{HbA}_{1 \mathrm{c}}$, baseline gap (except DCS), daily dose, drug adherence (except for DCS and CARDS), and eGFR (except for CARDS and Rotterdam Study). The $p$ value of the genotype effect is given. In the meta-analysis, a fixed-effects model was used

\begin{tabular}{llllll}
\hline Study & Group & $n$ & OR $(95 \%$ CI $)$ & SE & $p$ value \\
\hline DCS & Total group & 929 & $1.27(1.03,1.58)$ & 0.14 & 0.028 \\
& Monotherapy & 547 & $1.32(0.99,1.78)$ & 0.19 & 0.062 \\
\multirow{2}{*}{ Rotterdam Study } & Dual therapy & 382 & $1.20(0.87,1.66)$ & 0.19 & 0.26 \\
& Total group & 182 & $1.44(0.87,2.39)$ & 0.36 & 0.15 \\
& Monotherapy & 65 & $1.97(0.72,5.42)$ & 0.19 & 0.19 \\
CARDS & Dual therapy & 117 & $1.40(0.77,2.57)$ & 0.30 & 0.27 \\
& Total group & 254 & $1.03(0.68,1.57)$ & 0.21 & 0.86 \\
\multirow{4}{*}{ Meta-analysis } & Monotherapy & 81 & $1.50(0.76,2.95)$ & 0.34 & 0.23 \\
& Dual therapy & 173 & $0.82(0.46,1.46)$ & 0.29 & 0.51 \\
Meta-analysis including & Total group & 1,365 & $1.24(1.04,1.49)$ & 0.09 & 0.016 \\
GoDARTS & Monotherapy & 693 & $1.38(1.07,1.80)$ & 0.13 & 0.015 \\
& Dual therapy & 672 & $1.15(0.89,1.48)$ & 0.13 & 0.29 \\
& Total group & 1,965 & $1.21(1.05,1.38)$ & 0.07 & 0.008 \\
UKPDS & Monotherapy & 1,460 & $1.25(1.07,1.46)$ & 0.08 & 0.005 \\
& Dual therapy & 505 & $1.08(0.84,1.40)$ & 0.13 & 0.54 \\
& Total group & 1,113 & $1.37(1.10,1.72)$ & 0.11 & 0.006 \\
Meta-analysis & Monotherapy & 284 & $1.82(1.20,2.78)$ & 0.21 & 0.005 \\
& Dual therapy & 829 & $1.23(0.94,1.62)$ & 0.14 & 0.13 \\
& Total group & 4,443 & $1.25(1.13,1.38)$ & 0.05 & $7.8 \times 10^{-6}$ \\
& Monotherapy & 2,437 & $1.33(1.16,1.50)$ & 0.07 & $1.4 \times 10^{-5}$ \\
& Dual therapy & 2,006 & $1.15(0.99,1.34)$ & 0.08 & 0.067 \\
\hline
\end{tabular}


Fig. 1a, electronic supplementary material [ESM] Fig. 1). When the analysis was restricted to those with a baseline $\mathrm{HbA}_{1 \mathrm{c}}>7.0 \%$ (53 mmol $\left./ \mathrm{mol}\right)$, similar results were obtained (ESM Table 1). In the meta-analysis of the three novel cohorts where $\mathrm{HbA}_{1 \mathrm{c}}$ reduction was assessed as a quantitative trait, there was no significant effect of rs11212617 on $\mathrm{HbA}_{1 \mathrm{c}}$ reduction, partly because of the CARDS data being in the opposite direction to the other two cohorts, as reported previously (Fig. 1b). In the combined meta-analysis of all five replication cohorts from this and our previous study, the $\mathrm{C}$ allele was associated with an $\mathrm{HbA}_{1 \mathrm{c}}$ decrease of -0.050 $(95 \% \mathrm{CI}-0.089,-0.010)$ per copy of the $\mathrm{C}$ allele ( $p=0.013$; $I^{2}=59 \%, p=0.05 ; p_{\text {Egger }}=0.44$; Table 3 , Fig. $1 \mathrm{~b}$, ESM Fig. 1b). Again, when the analysis was restricted to those

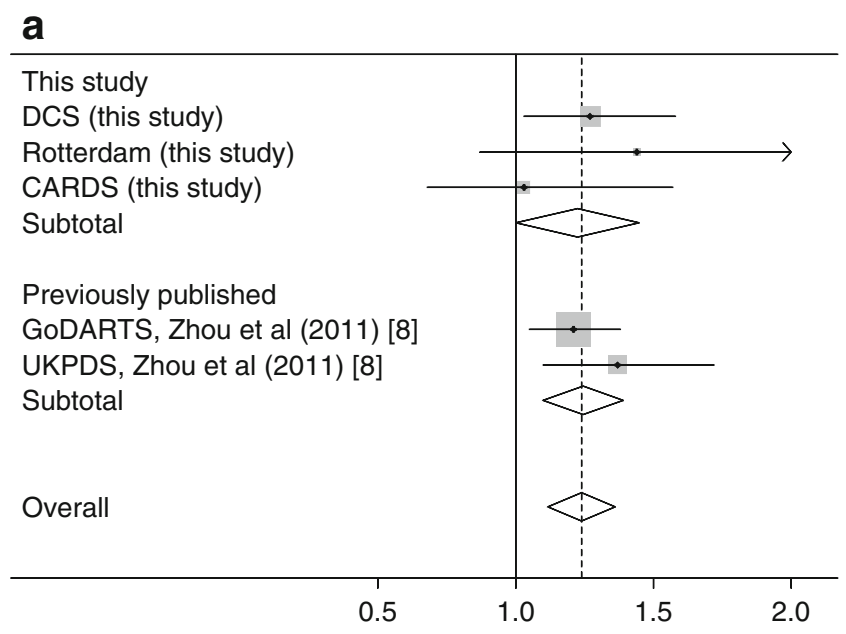

\section{b}

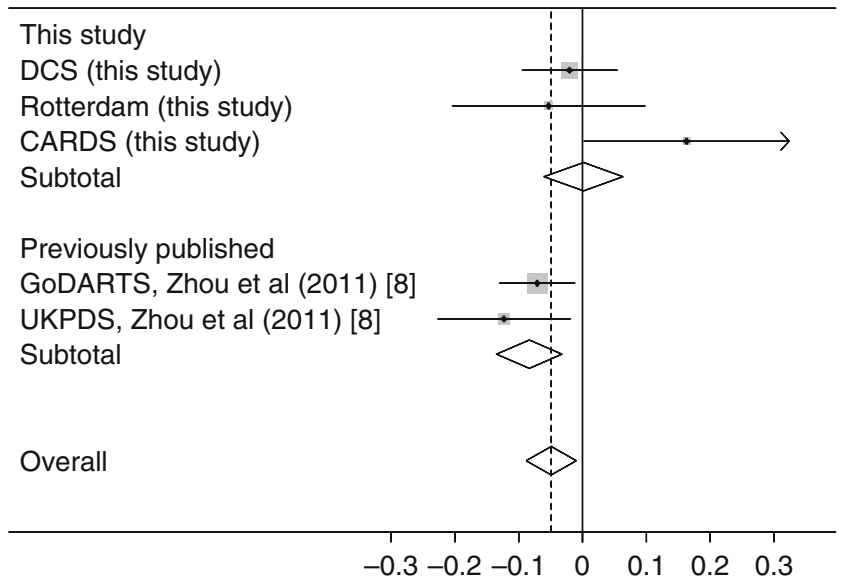

Fig. 1 Association between rs11212617, metformin treatment response and treatment $\mathrm{HbA}_{1 \mathrm{c}}$ in different cohorts. The grey squares and horizontal lines indicate (a) the cohort-specific ORs and 95\% CI for the ability to reach the treatment target of $\mathrm{HbA}_{1 \mathrm{c}} \leq 7 \%(53 \mathrm{mmol} /$ mol) and (b) the cohort-specific $\beta$-coefficients and $95 \%$ CI for the treatment $\mathrm{HbA}_{1 \mathrm{c}}$ as a continuous variable. The size of the squares is proportional to the weights of the studies. GoDARTS and UKPDS data were from Zhou et al [8] with a baseline $\mathrm{HbA}_{1 \mathrm{c}}$ above $7.0 \%(53 \mathrm{mmol} / \mathrm{mol})$, similar results were obtained (ESM Table 2).

\section{Discussion}

In this study, we show replication of the reported association between a SNP at a locus that includes the $A T M$ gene and glycaemic treatment success with metformin in the Dutch DCS cohort [8]. We noted a similar, although non-significant, trend in the smaller Rotterdam Study, but this trend was not observed in another small study, CARDS. Meta-analysis of data from these cohorts, which, despite intrinsic differences, provide clear, directionally consistent effects and shows replication of the previously reported association [8].

Importantly the effect of the SNP on metformin treatment success was observed in most cohorts, while there was no evidence for bias, and the characteristics of the cohorts differ, including data from population-based cohorts, prospective cohorts and clinical trials, indicating that the effect and its size are robust across European white populations. Furthermore, it is important to note that the association with treatment success is present regardless of baseline $\mathrm{HbA}_{1 \mathrm{c}}$, providing further evidence for the robustness of this observation.

The effect in the monotherapy group is larger than in the dual therapy group, as observed in the previous study. The underlying mechanism of this observation is unknown. As metformin is the recommended first-line therapy, patients receiving metformin monotherapy are probably earlier in their disease process. Accordingly, the observed difference between mono and dual therapy might be explained by the negative effects of prolonged disease duration on treatment efficacy. However, it might also be that sulfonylurea therapy antagonises some of metformin's effects mediated through mTOR-dependent and -independent signalling, as has been suggested recently by Wang et al [16]. Further research is required to elucidate the exact underlying mechanism.

The effect of the SNP on the ability to achieve $\mathrm{HbA}_{1 \mathrm{c}}$ $\leq 7 \%(53 \mathrm{mmol} / \mathrm{mol})$ is stronger than the quantitative $\mathrm{HbA}_{1 \mathrm{c}}$ reduction, yet the quantitative trait should have greater statistical power. One possible explanation for the 'treat-totarget' model appearing to be better is that the patients and clinicians aiming for a particular target may not intensify treatment further once it is achieved. The low baseline $\mathrm{HbA}_{1 \mathrm{c}}$ of $6.7 \%(50 \mathrm{mmol} / \mathrm{mol})$ in the DCS probably reflects increased awareness of diabetes among patients and caregivers and current prescribing practice, where metformin is introduced immediately after diagnosis and based on fasting glucose rather than $\mathrm{HbA}_{1 \mathrm{c}}$ [17]. This most likely explains the lack of a genotype effect on $\mathrm{HbA}_{1 \mathrm{c}}$ reduction in this cohort, as there is not much scope for the absolute $\mathrm{HbA}_{1 \mathrm{c}}$ to decrease on metformin initiation. 
We have shown, by additional replication and metaanalysis, that our initial report is consistent in multiple populations, supporting the finding that variation at the ATM locus is the most robust metformin response variant to date. Our data further support the notion that genetic variation does not only affect disease susceptibility, but also affects treatment response. Given the currently sparse efforts to elucidate the genetic architecture of type 2 diabetes treatment response, our data imply that future large-scale wellpowered studies might be successful in identifying further novel loci affecting treatment response. This is particularly pertinent for metformin response, where the biology of its working mechanism is not fully elucidated. It is clear that additional research is required to establish that rs11212617 is the causal SNP and that the ATM gene is the causal gene at this locus. In addition, it is necessary to investigate the mechanism whereby $A T M$ variation alters metformin response.

Acknowledgements We are grateful to all the participants in this study, to the general practitioners, to the Scottish School of Primary Care for their help in recruiting the participants, and to the whole team, which includes interviewers, computer and laboratory technicians, clerical workers, research scientists, volunteers, managers, receptionists and nurses.

Funding The work in this study was financially supported by the Dutch Diabetes Research Foundation grant 2006.00.060. The Wellcome Trust provides support for the Wellcome Trust UK Type 2 Diabetes Case Control Collection (GoDARTS) and informatics support is provided by the Chief Scientist Office. The Wellcome Trust funds the Scottish Health Informatics Programme, provides core support for the Wellcome Trust Centre for Human Genetics in Oxford, and funds the Wellcome Trust Case Control Consortium 2. This research was specifically funded by Diabetes UK (07/0003525). N. van Leeuwen is a member of the International Research Training Group (IRTG) funded by the DFG (GRH 1389/1) and NWO (grant DN95-420). K. Zhou holds a Henry Wellcome Post-Doctoral Fellowship.

Duality of interest R.R. Holman received research support from Amylin, Bayer, Merck and Novartis, attended advisory boards with Amylin, Lilly, Merck, Novartis and Novo Nordisk, and gave lectures supported by Bayer, Lilly Merck and Merck Serono. H. Colhoun received research support from Pfizer Inc., Roche Pharmaceuticals, Boehringer Ingelheim, Astrazeneca, Eli Lilly \& Company, attended advisory boards with sanofi-aventis, Pfizer Inc., Novartis Pharmaceuticals, Elis Lilly \& Company, received lecture fees from Pfizer Inc., and is a Stock/Shareholder of Roche Pharmaceuticals. The other authors declare that there is no duality of interest associated with this manuscript.

Contribution statement All the authors contributed to the conception and design of the study and critical revision of the manuscript and all authors gave final approval of the version to be published. NvL, LtH, EP and MB contributed to analysis and interpretation of the data.
Open Access This article is distributed under the terms of the Creative Commons Attribution License which permits any use, distribution, and reproduction in any medium, provided the original author(s) and the source are credited.

\section{References}

1. Nathan DM, Buse JB, Davidson MB et al (2009) Medical management of hyperglycaemia in type 2 diabetes: a consensus algorithm for the initiation and adjustment of therapy. A consensus statement from the American Diabetes Association and the European Association for the Study of Diabetes. Diabetologia 52:17-30

2. Owen MR, Doran E, Halestrap AP (2000) Evidence that metformin exerts its anti-diabetic effects through inhibition of complex 1 of the mitochondrial respiratory chain. Biochem J 348:607-614

3. El-Mir MY, Nogueira V, Fontaine E, Averet N, Rigoulet M, Leverve X (2000) Dimethylbiguanide inhibits cell respiration via an indirect effect targeted on the respiratory chain complex I. J Biol Chem 275:223-228

4. Zhou G, Myers R, Li Y et al (2001) Role of AMP-activated protein kinase in mechanism of metformin action. J Clin Invest 108:11671174

5. Hawley SA, Ross FA, Chevtzoff C et al (2010) Use of cells expressing gamma subunit variants to identify diverse mechanisms of AMPK activation. Cell Metab 11:554-565

6. Shaw RJ, Lamia KA, Vasquez D et al (2005) The kinase LKB1 mediates glucose homeostasis in liver and therapeutic effects of metformin. Science 310:1642-1646

7. Foretz M, Hebrard S, Leclerc J et al (2010) Metformin inhibits hepatic gluconeogenesis in mice independently of the LKB1/ AMPK pathway via a decrease in hepatic energy state. J Clin Invest 120:2355-2369

8. Zhou K, Bellenguez C, Spencer CC et al (2011) Common variants near ATM are associated with glycemic response to metformin in type 2 diabetes. Nat Genet 43:117-120

9. Fu X, Wan S, Lyu YL, Liu LF, Qi H (2008) Etoposide induces ATM-dependent mitochondrial biogenesis through AMPK activation. PLoS One 3:e2009

10. Suzuki A, Kusakai G, Kishimoto A et al (2004) IGF-1 phosphorylates AMPK-alpha subunit in ATM-dependent and LKB1independent manner. Biochem Biophys Res Commun 324:986-992

11. Florez JC, Jablonski K, Taylor A, Pollin T, Shudiner A, Diabetes Prevention Program Research Group (2011) The C allele of ATM rs11212617 does not associate with metformin response in the Diabetes Prevention Program. Diabetes 60:A386 (Abstract)

12. Welschen L (2008) Disease management for patients with type 2 diabetes: towards patient empowerment. Int J Integr Care 8:e69

13. Hofman A, van Duijn CM, Franco OH et al (2011) The Rotterdam Study: 2012 objectives and design update. Eur J Epidemiol 26:657-686

14. Colhoun HM, Betteridge DJ, Durrington PN et al (2004) Primary prevention of cardiovascular disease with atorvastatin in type 2 diabetes in the Collaborative Atorvastatin Diabetes Study (CARDS): multicentre randomised placebo-controlled trial. Lancet 364:685-696

15. Richards JB, Rivadeneira F, Inouye M et al (2008) Bone mineral density, osteoporosis, and osteoporotic fractures: a genome-wide association study. Lancet 371:1505-1512

16. Wang Q, Cai Y, Van de Casteele M, Pipeleers D, Ling Z (2011) Interaction of glibenclamide and metformin at the level of translation in pancreatic beta cells. J Endocrinol 208:161-169

17. American Diabetes Association (2011) Standards of medical care in diabetes: 2011. Diabetes Care 34(Suppl 1):S11-S61 